\title{
Mobile Application for Noise Pollution Monitoring through Gamification Techniques
}

\author{
Irene Garcia Martí, Luis E. Rodríguez, Mauricia Benedito, Sergi Trilles, \\ Arturo Beltrán, Laura Díaz, and Joaquín Huerta
}

Institute of New Imaging Technologies (INIT), University Jaume I (UJI)

\{irene.garcia, pupo, mauri.benedito, sergi.trilles, arturo.beltran, laura.diaz, huerta\}@uji.es

http://www.geoinfo.uji.es

\begin{abstract}
Full data coverage of urban environments is crucial to monitor the status of the area to detect, for instance, trends and detrimental environmental changes. Collecting observations related to environmental factors such as noise pollution in urban environments through classical approaches implies the deployment of Sensor Networks. The cost of deployment and maintenance of such infrastructure might be relatively high for local and regional governments. On the other hand recent mass-market mobile devices such as smartphones are full of sensors. For instance, it is possible to perform measurements of noise through its microphone. Therefore they become low-cost measuring devices that many citizens have in their pocket. In this paper we present an approach for gathering noise pollution data by using mobile applications. The applications are designed following gamification techniques to encourage users to participate using their personal smartphones. In this way the users are involved in taking and sharing noise pollution measurements in their cities that other stakeholders can use in their analysis and decision making processes.
\end{abstract}

Keywords: Gamification, mobile applications, environmental monitoring, noise pollution, PPGIS, VGI.

\section{Introduction}

Many people on Earth are living in cities and it is expected that $70 \%$ of human population will live in cities by 2050 [1]. This fact influences in the growth of activities carried on in industrial parks and urban areas including traffic and other human activities that affect environmental conditions such as noise pollution.

In order to guarantee a sustainable development of our cities, European policies try to regulate the evolution of these environmental conditions. Among others, the European Noise Directive (END) [2], adopted in 2002, establishes a legal framework related to the assessment of environmental noise. In Article 3, it is defined "environmental noise" as "unwanted or harmful outdoor sound created by human activities, including noise emitted by means of transport, road traffic, rail traffic, air traffic, and from sites of industrial activity". In the same article, other concepts like "annoyance" or "harmful effects for health" are defined. 
There are authors who state that noise pollution could seriously affect human health: In $[12,13]$ it is discussed the set of issues a sustained exposure to noise pollution could trigger, ranging from less-severe sleep disturbances or hearing impairment to much serious cardiovascular disturbances or mental instability.

Daily city activities cannot be stopped, but it is important to continuously pinpoint noise observations to understand if there is any underlying problem that could be a cause of human health issues that should be solved by any government entity. Noise pollution could be measured through sensor networks, but it is a phenomenon that occurs in the entire city with different intensity throughout time and space. Therefore, data acquired with this method could not be enough to depict the city situation.

In this context, it is important to consider a different way for data collection with a high temporal and spatial noise data resolution and with a low deploying cost. The key for this noise pollution monitoring in cities is based in citizenship participation. Involving citizens in monitor noise pollution by using mobile applications is a very cheap method for administrations to acquire real-time data that may help local or regional governments to identify potential problems and therefore, try to solve them.

In this paper we present two mobile application prototypes to collect noise pollution data in urban areas. One of the issues that arise when developing a crowdsourcing monitoring application is the fact that we rely on the measurements taken by the users, but, how to engage users to use this application and provide observations? The main idea is to engage citizens to take noise samples by gamifying the process of acquiring data to obtaining a noise pollution map. One of the problems we identify in the traditional mobile noise measurement taking is that we have to convince the user he or she has to start taking noise samples and keep this practice through time. In this new data acquiring approach, it is crucial the massive and maintained participation over time from general public. This would help creating a huge noise pollution repository to be used for further and more accurate analysis. The difficulty arises from the boredom of doing repetitive tasks without an incentive or amusing elements.

Here is where gamification techniques take major importance, because they provide a mechanism to motivate users to use the application and provide information while they are using a gamified application. In this paper we propose a general approach for noise pollution data collection that implements a crowdsourcing noise pollution monitoring application based on gamification techniques. The main idea applying these techniques to environmental monitoring is to encourage users to participate in data collection process by using their personal smartphone devices. The output of this public participation is a high amount of georeferenced noise pollution dataset that can be later used for further analysis or decision making processes. We demonstrate the application of these gamification techniques by implementing two mobile application prototypes as a proof of concept. Therefore, NoiseBattle and NoiseQuest mobile applications will be presented as a new participatory way of noise pollution data collection.

The remainder of this paper is organized as follows: In section 2 we discuss the background of this work, including topics such as noise pollution monitoring, general gamification concepts and related work. In section 3 we describe how we applied to this project the gamification concepts. In section 4 we briefly describe the general architecture of this Project and present the two prototype applications in development and finally, in section 5 , we present the conclusions and future work ideas. 


\section{Background and Related Work}

\subsection{Environmental Noise Pollution Monitoring}

Environmental monitoring is a crucial task to control our planet status and development trends. With the adequate set of technologies and tools it is possible to monitor almost any measurable parameter. In our case, we are interested in controlling the noise levels on cities.

In [3] it is described and applied the concept of creating a GI system with Volunteered Geographic Information (VGI), called Public Participatory GIS. The authors define a PPGIS as the result of using GIS capabilities and techniques by the general public, although it was first described in [4]. Moreover, in this paper, scientist community is encouraged to break top-down strategies of delivering geographic data, where government entities, institutions or companies provide them at their own interest. The authors' state that is important to create new bottom-up data productions in order to create GI systems that really fits users' needs and availability. An example of PPGIS application it is found in [3], where Canela (Brazil) users can post comments about POIs related to health or education, such as complains for a school menu service or asking for information related to cultural heritage.

In [5] we could find a good description of the concept "Citizens as Voluntary Sensors". The author states that humanity as a collective, possesses a huge amount of knowledge about the Earth surface and its properties, such as place names, topographic areas or status of a transportation network. If we enable this people with electronic devices to digitize this information, we have a massive collection of raw data collected by volunteers that we can use in our analysis, services and geoprocesses.

Following the PPGIS and Citizens as Voluntary Sensors guidelines, we thought that it would be interesting to join both concepts to apply them in environmental noise pollution monitoring. In this field, some attempts already exist:

According to [6] there are three main approaches to assess noise pollution: simulation maps and data collection through sensor networks. First method consists in applying physical noise propagation laws considering well-known noise sources to get noise affection maps, while second method is based on acquiring data using a distributed network of sensor devices. Finally, there is another method they outline, based in the direct participation of citizenship by providing VGI.

In this paper, we are going to extend the idea described in [6] by developing two mobile applications to collect noise pollution data in urban areas by applying to its development Gamification concepts. Therefore, as users will collect their own noise samples, we are using the concept of citizens as sensors [5] and, as we are going to publish our data in a Noise Mapping Platform, we are contributing to the enlargement of a PPGIS.

\subsection{General Gamification Concepts}

The 'citizens as sensors' paradigm [5] implements the idea of crowdsourcing the sensor data collection. This is a straightforward way of collecting huge amount of data at 
a very low cost. Nevertheless, users need an incentive to make volunteers collect those data willingly. At this point here is where the gamification methodology plays its role. In [7] it is questioned how the general public can be motivated to voluntary participation by displaying noise data publicly on a Web GIS platform through maps or allowing tagging the noises measured to enrich this information in a subjective way. However, applications with no incentives will probably attract users highly motivated to collaborate with this platform, just for their satisfaction of contributing to science. Those users will gladly update their observations in the long term, but this set of noise data, although big, will not represent the entire city situation. Gamifying a repetitive or complex problem breaks this gap/wall between a scientific problem and citizenship and allows any citizen to contribute to science unconsciously.

The core concept for gamifying is engagement. As seen in [8], engaging users to do something is crucial to encourage citizenship to collaborate with a project. Engagement could be achieved by motivating people, so it is important to create nice and user-friendly applications that foster people to take the measurements we need.

According to [8] to do a good gamification process it is important to consider four key concepts: user status, access, power \& stuff.

Status: Users usually like to compare or share their progress and achievements with other users, to see who is in a better or worse position than others. To do this, it is necessary to split game progress in stages or levels, from easiest to hardest ones.

Access: This concept encourages allowing users to unlock new features depending on their contribution or participation in the game. It is important to make these features exclusive enough to engage the user who achieves them more attached to the application.

Power: With the transference of some power to some users, it is possible to encourage them to keep using the application. The power can be represented by letting them to do actions that are not allowed to users who have used less the application.

Stuff: In addition to all functional concepts stated above, it is important to provide a set of free rewards, badges or gifts for users as an incentive to keep playing. Those items are attractive to users because they can make the difference with others.

\subsection{Related Work}

Nowadays, there are many successful examples of gamified and purpose-oriented applications. It is the case of Phylo ${ }^{1}$ an application that exploits the natural capacity of human being to recognize visual patterns, an operation with a high computational cost. Phylo presents DNA sequences in the mobile device screen in a gamified context and engages users preparing a race competition among them and providing awards and acknowledgment for winners.

Another example of gamification comes from automobiles' brand Nissan ${ }^{2}$. It includes in their new car models an application for monitoring gas emissions and featuring an automatic gaming system to provide real-time information about the gases

\footnotetext{
http://news.cnet.com/8301-17938_105-20024075-1.htm

http://news.cnet.com/8301-17938_105-20026662-1.html
} 
emitted to the atmosphere. Users are then encouraged to drive responsibly to go up in drivers' rankings.

Regarding the collection of noise using public participation, several projects have been carried on. For example, in [15] is presented a project where the users can contribute to collect noise from the city and publish it in the NoiseTube platform. The platform offers a mobile application that consumes an API for sending the collected data. Later the user can see the maps with the noise data in a website. Other project worth to mention is presented in [14]. In this case the authors describes a mobile application for collecting noise data, and add the possibility of including extra information related with the perception of the noise measured (i.e. annoyance) or the location (i.e. home or work place). In both cases the incentive for collecting data is the mere contribution.

Finally, it is also important to consider the gamification techniques applied to produce FourSquare application, as it is a successful social game oriented to businesspurposes that breaks the wall between the virtual/real world by providing physical rewards to users.

\section{Applying Gamification Concepts}

After explaining the gamification theoretical concepts in section 2.2, we are going to explain how we carried on the gamification process in particular to our prototypes. Considering the two use cases we have developed, those concepts have been applied as follows:

Status: In our project, we have created this progress based on the number of noise observations: as users collect information, they get more points that will increase general score and therefore, their level.

Access: The access concept is grounded as unlocking quests or owning more city areas, depending on the prototype. Collecting noise observations is the key to progress in both applications. Data acquisition is rewarded: as long as the users collect more noise observations, access to different kind of things is granted.

Power: In one of the prototypes we provide power by giving the capability of sending noises (or sounds) to an enemy in the battle. This kind of actions are important to foster competition among users, so they feel motivated to keep progressing in the game to gain that special power another user has.

Stuff: A set of avatars and rewards have been created for both use cases.

Regarding the target users analysis, we followed the classification found in [9]. In this paper, the author divides users in four different types: killers, achievers, socializers \& explorers. In our prototypes, we targeted the following users:

Noise Battle: The user profile we targeted is mainly Achiever. In this application, the idea is providing an environment where the user can win points and conquer areas, with the purpose to make the player feel rewarded just by winning and sending noises to the enemies. The competition factor is very important to make their achievements more satisfying. 
Noise Quest: The type of user targeted in this second case is Explorer. Here the competition with other users is not as intense as the previous case. In this application it is more important walking around the city taking measurements than the score achieved by the number of observations taken. The "goodness" of the observation is more valuable than the number of measurements collected.

\section{$4 \quad$ Architecture and Prototypes}

\subsection{General Architecture}

This section describes in detail the conceptual architecture we used. Figure 1 depicts the general architecture of the project. The prototypes in-development for this project follows the same schema. As seen, the modules are divided in three parts: Mobile Client-side application, Middleware Layer and Remote Server-side that are described below these lines.

The Mobile application provides the functionality that allows user to take measurements, send them to the middleware and check the general progress of the battle in course. We suggest that the application should be divided in the following modules: User Interface, Measurement, Mapping and Connection \& Encoding. For the User Interface we used Android SDK because it is a free and growing development platform and provides a good integration with Google Maps, the engine we used for our Mapping module. Regarding the Connection \& Encoding Module, we decided to implement our own classes in Java to connect and send the data to the Middleware Server. For the Measurement Module we used part of the mobile noise pollution measurement application developed by IFGI (Institute for Geoinformatics, University of Münster) called NoiseDroid. NoiseDroid is an open-source mobile application that allows the user taking noise samples. This application has its own noise quality assessment system which provides information about the usefulness of the noise data collected $[10,11]$. Due to the open-source condition of NoiseDroid, we reused source code related to the collection and assessment of the noise samples, and include it in our Measurement Module.

Middleware layer has as main goal to attend all incoming noise observations, store them in a noise database and send the measurements taken to a remote server. This layer, that should lay on an intermediate server, takes care as well of keeping the user's status up-to-date in real-time, writing in the database the new data and sending back the status to the user while notifying other users of the changes on the game they are playing. We have divided the functionality of this server in three different modules: Client Communication, Transactional, Remote Server Connection. For the Client Communication Module we decided to develop our own set of classes in Java. The Transactional Module is programmed in Java as well, but we used Hibernate for the communication with the database. In the case of the Remote Server Connection Module we used the API provided by the server for sending the noise data.

Remote Server-side is the module that will contain all retrieved VGI noise data from users and will allow the analysis by general public by providing some mapping layers. We think about the functionality of this server in these modules: Mapping and 
Data Management. In order to reuse available resources, we decided to contribute to Open Noise Map Platform (ONMP) by sending our collected data to this noise mapping platform. ONMP was developed by IFGI and provides a Web GIS interface where it is possible to see uploaded noise measurements comfortably, using a map layout.

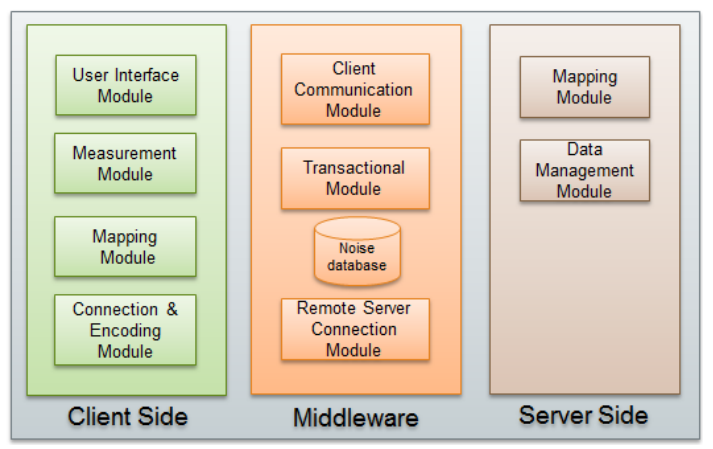

Fig. 1. General architecture of the proposed solution

\subsection{Noise Battle Prototype}

Noise Battle is a gamified mobile application developed for Android platform where users have to move around a city taking noise samples. Currently the game is on development and it is not entirely functional or available to download. The final goal of this game from the user's point of view is conquering the city by taking noise measurements.

The city is split into cells of a grid, so the user can conquer the cells by taking more and better measurements than other users in the area. During the game, the users are rewarded by different means: either by measuring in the proximity of one of the rewards placed in the grid or by conquering some cell of it. The rewards might include the possibility of sending noises or sounds to the foes. The sounds that can be sent to the rivals are used to show the power obtained by the sender of the noise. The rivals have the option of re-conquering previously conquered cells by performing better quality measurements or more recent measurements. These mechanisms should encourage the players to provide more accurate (based on a criteria taken from the API used) or updated noise data. Regarding the rewards, we have considered awarding measurements dispersion and data quality (based on NoiseDroid quality assessment) in order to assure a regular number of observations taken in the entire city area. Besides, we also took into account to place rewards (or having higher density of them) in zones where there is more interest about noise pollution conditions. The observations gathered are immediately uploaded to the middleware server, which further submit it to the ONMP platform. The ONMP platform can store it and make it available for visualization or processing.

In Figure 2 (left) it is possible to see a sample of the battlefield where a game is going to start. As seen, the city is divided in a grid where each cell represents an area the 
user can conquer through taking noise measures. For each measurement performed, it is possible to see the minimum, maximum and mean of noise measured. Then the player can decide to submit it as his move in the game. In this case there are some items placed for each cell that represent locations where the player can take a noise measurement and receive a reward. Figure 2 (centre) represents a more advanced state of the battle for that city, where some users have started using the application and conquered some areas. The areas conquered can be recognized by the colour of the cell. In this scenario there are three players, each of them with a different colour and avatar associated competing each other. Figure 2 (right) provides a higher level of detail of the image in the centre. In the next section we briefly describe the second prototype.
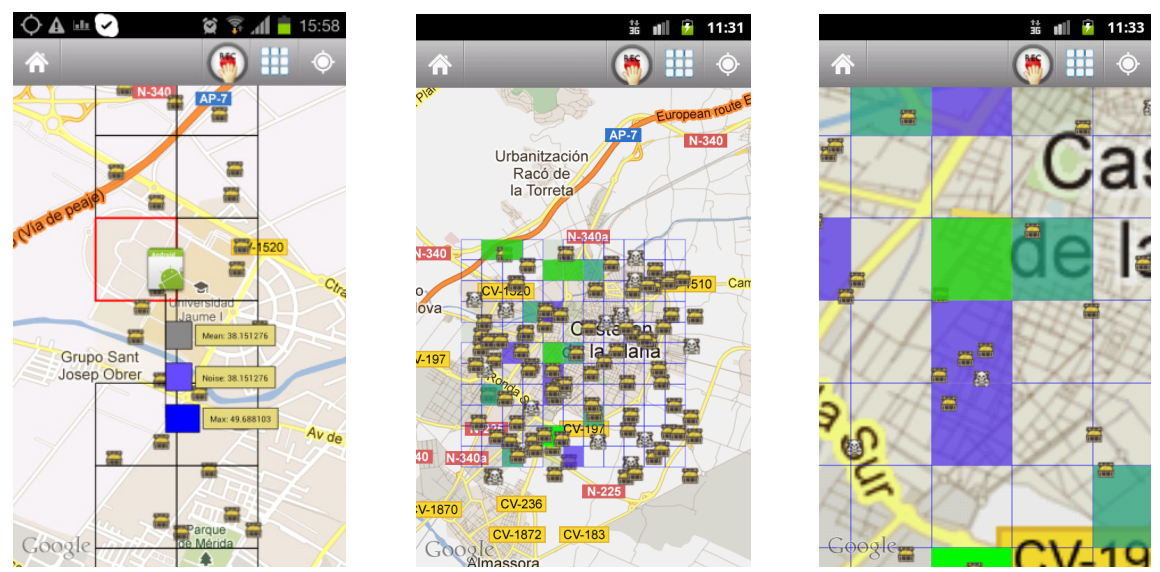

Fig. 2. Some images depicting NoiseBattle prototype working

\subsection{Noise Quest Prototype}

Noise Quest is a prototype of mobile application for Android platform that using quests, challenges and missions will guide a user around a city taking noise samples following a storyline. At this point, NoiseQuest application development is in a very early stage, so it is not possible to present any sample screenshot. The quests are focused in conquering different POIs by taking samples, making a competition with the different users playing the game and progressing in the story. The general idea of this application is to obtain noise measurements distributed uniformly around the city throughout time. For this purpose, the application will guide the user over all city area following a storyline where he or she is the main character. This game has different levels of difficulty so quests will be easier at the very beginning of the game and getting harder as long as the player advances in the game. The game starts in the user position and taking this point as a centre it draws three concentric circles with a determined radius whose size depends on the selected level: the higher the level, the bigger the circles are and therefore, the general difficulty of the game increases until covering the entire city area. First quests consist in obtaining measurements in the 
user's surroundings, located in the most inner circle. As long as the user completes the quests, the missions and challenges will lead the user to positions farther away, in the outer circles.

\section{Conclusion}

In this paper we presented an approach for designing environmental noise pollution monitoring applications based on crowdsourcing by means of applying gamification techniques. We presented a conceptual architecture and components to implement this kind of applications. For demonstration purposes two gamified mobile applications for noise pollution monitoring have been developed. The use of these applications by general public could contribute to increase urban society welfare through noise sampling, locating areas with a higher noise pollution to make some research or analysis and try to solve the problem.

Gamifying it is an interesting process to convert tiring and repetitive tasks in others lighter and more user-engaging ones that can provide huge amount of data to alllevels government entities and other decision makers. Logically, if a tool capable of spotting noise problems is created, it would be interesting from local authorities to consider this potential source of data to improve liveability of a city.

Developing purpose-oriented and gamified applications for mobile platforms it is very interesting due to its big range of possibilities and topics to create applications. Nevertheless, we think that in parallel, it is recommended to provide a pure GIS application where it would be possible to check and make operations with the set of data and make analysis.

As future work, it would be interesting to implement mechanisms for filtering and correcting VGI data, as per nature, some of them might be false or inaccurate for scientific analysis. On the prototypes functionality, two improvements could be done: at this point, both applications are spatially dividing the city in a grid in NoiseBattle case and in concentric and growing circles in NoiseQuest example. It would be interesting to change these geometric shapes into more real ones that fit a city district or neighborhood, so the user is competing in more natural divisions. The second idea would be implementing a noise measurements expiring policy for observations. A user would lose his/her score progressively in time with the aim of sticking the user to the application: the idea is encouraging them to keep them taking noise samples by slowly removing their conquers and score due to inactivity.

Acknowledgements. This work has been partially supported by the GEOCLOUD project ref. IPT-430000-2010-11, INNPACTO 2010 subprogramme, Ministry of Science and Innovation; OSMOSIS: Open Source Mobile Sensor Information System. IMPIVA Institute (Institute of Small and Medium-sized Enterprises from Generalitat Valenciana) and European Regional Development Fund (ERDF), ref. IMPIVA I+D IMIDTA/2009/793 and IMIDTA/2010/24. 


\section{References}

1. United Nations: Report about State of the World's Cities 2010/2011: Bridging the urban divide (2011)

2. European Parliament: Directive 2002/49/EC of the European Parliament and of the Council of 25 June 2002 relating to the assessment and management of environmental noise (2002)

3. Bugs, G., Granell, C., Fonts, O., Huerta, J., Painho, M.: An assessment of Public Participation GIS and Web 2.0 technologies in urban planning practice in Canela, Brazil. Cities 27(3), 172-181 (2010)

4. Nyerges, T., Barndt, M., Brooks, K.: Public participation geographic information systems. In: Proceedings of Auto-Carto 13, Seattle, WA, American Congress on surveying and mapping. Bethesda, MD, pp. 224-233 (1997)

5. Goodchild, M.: Citizens as Voluntary sensors: spatial data infrastructure in the world of Web 2.0. International Journal of Spatial Data Infrastructures Research 2, 24-32 (2007)

6. Maisonneuve, N., Stevens, M., Ochab, B.: Participatory noise pollution monitoring using mobile phones. Information Polity 2010 15, 51-71 (2010)

7. Maisonneuve, N., Stevens, M., Niessen, M., Hanappe, P., Steels, L.: Citizen Noise Pollution Monitoring. In: Proceedings of the $10^{\text {th }}$ International Digital Government Research Conference 2009 (2009)

8. Zichermann, G., Cunningham, C.: Gamification by Design. Implementing Game Mechanics in Web and Mobile Appls. O'Reilly Media, Inc. (2011)

9. Richard, B.: Hearts, clubs, diamonds, spades: Players who suits MUDs (1996), http: / / www. mud.co.uk/richard/hcds.htm

10. Foerster, T., Jirka, S., et al.: Integrating Human Observations and Sensor Observations the Example of a Noise Mapping Community. In: Proceedings of Towards Digital Earth Workshop at Future Internet Symposium. Berlin, Germany, September 2010; CEUR-WS: Aachen, Germany, vol. 640 (2010)

11. Everding, T., Jürrens, E., Andrae, S.: In-stream Validation of Measurements with OGC SWE Web Services. In: Second International Conference on Advanced Geographic Information Systems, Applications and Services, pp. 93-98. IEEE Computer Society (2010)

12. Goines, L., Hagler, L.: Noise Pollution: A modern plague. Southern Medical Journal 100, 287-294 (2007)

13. Trombetta Zannin, P.H., BelisárioDiniz, F., Alves Barbosa, W.: Environmental noise pollution in the city of Curitiba, Brazil. Journal of Applied Acoustics 63, 351-358 (2002)

14. Bilandzic, M., Banholzer, M., Peev, D., Georgiev, V., Balagtas-Fernandez, F., De Luca, A.: Laermometer - A Mobile Noise Mapping Application. In: Proceedings of NordiCHI 2008 (2008)

15. Maisonneuve, N., Stevens, M., Niessen, M.E.: NoiseTube: Measuring and mapping noise pollution with mobile phones. In: Proceedings of the $4^{\text {th }}$ International ICSC Symposium, Information Technologies in Environtmental Engineering (ITEE 2009), Thessaloniki, Greece, May 28-29 (2009)

16. D'Hondt, E., Stevens, M., Jacobs, A.: Participatory noise mapping works! An evaluation of participatory sensing as an alternative to standard techniques for environmental monitoring 